\title{
A PSO based Information Hiding Approach using Pixel Mapping and Wavelet Transformation
}

\author{
Tanmoy Halder ${ }^{\# 1}$ \\ \# 1 Assistant Professor, Dr.B.C.Roy Engineering College, Durgapur, India. \\ tanmoyhalder@gmail.com \\ Sunil Karforma*2 \\ *2 Professor, Dept. Of Computer Science, The University of Burdwan, Burdwan, India. \\ sunilkarforma@yahoo.com
}

\begin{abstract}
In this paper a new data hiding approach has been proposed combining Pixel value difference (PVD), Particle swarm optimization (PSO) and pixel mapping. The aim of this paper is to map the secret information within most suitable block of pixels to ensure minimum change. Each pixel groups are different in nature and may not map with any group of secret data. Appropriate mapping of pixels is conducted by verifying some parameters. Collective forms of those parameters create the fitness function. The fitness function of particles is chosen such a way that a balanced ratio between capacity and Signal to Noise Ratio(SNR)is to be maintained. To elevate security level of encryption, overlooking the spatial domain embedding Haar discrete wavelet transformation (DWT) has been used. Embedded images are compared and analysed against existing approaches in term of hiding capacity and induced noise.
\end{abstract}

Keywords: Steganography, Haar wavelet transformation, Pixel value difference, Particle swarm optimization, Structural similarity index.

\section{INTRODUCTION}

Steganography, the art of hiding secret message within a cover file is essential for secret digital communication. Recent advancement of Smartphone has increased sharing of digital files like documents, pictures, sound, videos etc.. Along with the advancement in communication technology the security of those important documents is also under threat. Digital Steganography is the simplest and efficient way in order to protect file sharing through digital media.

Since 2001 steganography has travelled a long way of advancement and improvement. Complex algorithms and approaches have been used to make it more robust and secure. Capacity and presence of noise in the stego image are two main challenging contradict issues for all those algorithms as high capacity approaches are always susceptible to noise. Steganography algorithms are mainly divided in two domains. 1) Spatial domain 2) Frequency domain. In spatial domain raw pixels are altered to hide secret message whereas in Frequency domain the pixels are converted to frequency. Frequency values are used to hide information. Spatial domain methods are always known for great hiding capacity when frequency domain methods are more secure against detection.

The best-known image steganographic method in spatial domain is the Least Significant Bit (LSB) replacement $[1,2,3]$. LSB bits of the pixel value are used to hide secret data.

Other than LSB methods Wu and Tsai [4] proposed a method known as Pixel value difference(PVD). In PVD non-overlapping block of two pixels are chosen from the cover image. Difference between the pixels justifies the amount of data to be hidden within those pixels. Ko-Chin Chang et al [5] proposed Tri-way pixelvalue-difference method. Here blocks of four pixels are chosen to create three pairs. Khodaei and Faez [6] proposed another algorithm based on PVD method. Here non-overlapping block of three pixels are chosen in raster scan order. In the middle pixel 3-LSB bits are altered with secret bits, therefore OPAP is applied on that pixel to reduce embedding error. Chi-Shiang Chan et al. [7] therefore extended Khodaei and Faez's work for flexible number of blocks. Block of size $\mathrm{M}^{*} \mathrm{~N}$ could be selected, where value of $\mathrm{M}$ and $\mathrm{N}$ vary from 1 to 7 . T.Halder \& S.Karforma[8] extended Chi-Shing Chan's method to increase the capacity of blocks. They proposed that, rather than increasing the size of blocks by just modifying the embedding algorithm capacity could be increased. 
Wang et al. [9] and Khodaei and Faez [10] introduced a genetic algorithm based approach using a substitution table while hiding secret bits. LSB embedding technique was used for embedding secret bits. P.Bedi and R Bansal [11] applied PSO to find the best area within cover image, thereafter LSB technique was used to embed secret information.. F.Heriniaina, and j.Sang. [12] applied PSO with already existing LSB Steganography in order to search suitable area within an image for hiding secret bits. PSO based approaches are always proven as better steganographic technique rather than non-PSO based techniques.

P.Bedi and R Bansal[13] proposed another approach using PSO. Structural Similarity Index(SSIM) and $\mathrm{BER}$ (Bit Error ratio) is used to form the objective function for a particle. A block of pixels was considered as a particle. Each particle was given a fixed number of bits to embed using LSB. Bedi and Bansal's approach did well with better PSNR value compared with other PSO based approaches.

Gulve and Joshi[14] proposed that using block wise embedding security level could be increased. $2 \times 3$ block of non-overlapping pixels were chosen and therefore PVD method applied to hide secret message.

Ghasemi et al.[15] applied Discrete Wavelet Transformation and Genetic Algorithm(GA) to increase security in existing methods. Wavelet Transformation is used to decompose the image in subbands whereas GA and Optical Pixel Adjustment Process(OPAP) applied to map secret data within cover image.

Al-Asmari et al.[16] proposed another Wavelet Transformation based method where message embedded using LSB replacement and PVD method. LL subband is used for LSB embedding and HH,HL,LH subbands are embedded with PVD method.

Gulve et al. [17] proposed another PVD based embedding algorithm with increase capacity. In the method the cover is divided into subbands using Haar Wavelet Transformation and all the four subbands LL,HL,LH and HH are utilised for embedding secret data. $2 \times 2$ block of pixels is chosen and therefore secret data is embedded using PVD method.

Fan $\mathrm{Li}$ et al.[18] proposed embedding using interleaving prediction in Haar-Wavelet based steganography. Rather than using sequential order zigzag order is used for scanning, therefore HH,HL and LH subbands are utilised for embedding. Prediction error between two consecutive values determines data embedding areas.

In order to reduce noise and to increase capacity the proposed approach introduced a hybrid technique combining PVD,LSB and Haar Discrete Wavelet Transformation. PSO applied to map secret block of bits in appropriate position. Each $3 \times 3$ block of frequency values have different capacity. Flexible set of secret data are mapped and adjusted within appropriate area for embedding. HH,HL and LH subbands are used for embedding. As high capacity blocks may incorporate more noise and very low capacity blocks may have high PSNR. Fitness function of PSO maintains a balance between noise and capacity of each block. While setting the Fitness function PSNR is used to ensure better quality stego image.

Rest of this paper is organized as follows: Section II gives idea about PVD method, section III discuss about PSO, in section IV Haar Wavelet Transformation is discussed in brief. Section V is about proposed work, Section VI contains experimented result and analysis of results is done in section VII followed by conclusion in section VIII.

\section{PIXEL VALUE DIFFERENCE}

The Pixel value difference(PVD) method proposed by Wu and Tsai[4]. In a greyscale image each pixel has a colour intensity value lies between 0 and 255. If the gap between two neighbour pixel value is high the region is a sharp area, more number of secret bits could be embedded within that difference. If the same gap is negligible then few bits could be embedded. Following this approach, PVD technique can adjust exact number of secret bits within gaps. The process is as follows:

$P$ and $Q$ are two neighbour pixels, the difference $(D)$ between $P$ and $Q$ is calculated as :

$D=|P-Q|$

Value of $D$ lies between $0 \& 255$. In a smooth region value of $D$ is less and in a sharp-edge region value of $D$ is high. Depending on the value of $D$, number of bits to be embedded is decided. $D$ is categorized according to the range table $R_{i}(\mathrm{i}=1,2 \ldots \ldots \mathrm{n})$. Range table decides how many bits could be embedded. Suppose the ranges are $R_{I}(0 . .7) R_{2}(8 . .15) R_{3}(16 . .31) R_{4}(32 . .61) R_{5}(62 . .123) R_{6}(124 . .255)$. Each range has a lower and upper limit says $L_{i}$ and $U_{i}$. For example for $R_{l}, L_{i}=0$ and $U_{i}=7$. Width $\left(W_{i}\right)$ of the range is a power of two and is calculated as

$W_{i}=\left(U_{i}-L_{i}+1\right)$

There after hiding capacity $\left(T_{i}\right)$ is calculated as

$T_{i}=\left\lfloor\log W_{i}\right\rfloor$ 
Here $T_{i}$ is the number of bits to be embedded as secret message. Bits from secret message are converted into decimal number $\left(T_{i}^{\prime}\right)$. The new difference between the pixels $P$ and $Q$ is calculated as

$$
D^{\prime}=L_{i}+T_{i}^{\prime}
$$

The secret data could be hidden by adjusting $P$ and $Q$ as $P^{\prime}$ and $Q^{\prime}$, the adjustment is as follows.

$$
P^{\prime}, Q^{\prime}=\left\{\begin{array}{l}
\left(P+\left\lceil\frac{m}{2}\right\rceil, Q-\left\lfloor\frac{m}{2}\right\rfloor\right) \text { if } P \geq Q \text { and } D^{\prime}>D \\
\left(P-\left\lfloor\frac{m}{2}\right\rfloor, Q+\left\lceil\frac{m}{2}\right\rceil\right) \text { if } P<Q \text { and } D^{\prime}>D \\
\left(P-\left\lceil\frac{m}{2}\right\rceil, Q+\left\lfloor\frac{m}{2}\right\rfloor\right) \text { if } P \geq Q \text { and } D^{\prime} \leq D \\
\left(P+\left\lceil\frac{m}{2}\right\rceil, Q-\left\lfloor\frac{m}{2}\right\rfloor\right) \text { if } P<Q \text { and } D^{\prime} \leq D
\end{array}\right\}
$$

Where $m=\left|D^{\prime}-D\right|$

\section{PARTICLE SWARM OPTIMIZATION}

Particle swarm optimization(PSO)[19] is a stochastic optimization technique developed by Kennedy,J., Eberhart,R.(1995). The idea of PSO is taken from Swarm intelligence which is inspired by social swarming behaviour of bird flocking, fish schooling or even in human social behaviour. PSO simulates the behaviours of bird flocking. Suppose, a group of birds is looking for food within a particular area in random manner. Food is available only within some selected area. A few of those birds found the food while all other birds are unaware about it. At each step other birds upgrade their movement towards the food by following those birds. Following this concept all birds succeed to reach at the destination where the food is available after completing some steps.

Inspired from the scenario, PSO use this concept to solve the optimization problems in different situation. In PSO, each single position is a "bird" and the search space is a "particle". Each particle has a fitness values which are evaluated by the fitness function. Velocity directs the movement of the particles. The particles fly or move through the problem space by following the current optimum particles.

The problem initialized with a group of random particles (solutions). In every iteration, each particle is updated by following two parameters. The first one is the best solution(fitness) it has achieved so far. It is evaluated by sorting and choosing the personal best fitness value. This value is called the pbest. Another best value that is tracked by the particle swarm optimizer is the best value obtained so far by any particle within a generation. It is called gbest. When a particle takes part of the population as its topological neighbours, the best value is a local best and is called the lbest.

After finding the two best values, the particle updates its velocity and positions, using the following equation 6 and 7.

$$
v[i]=v[i]+w i * c 1 * \operatorname{rand}() *(\text { pbest-current_solution }[i])+c 2 * \operatorname{rand}() *(\text { gbest-current_solution }[i])
$$

$$
\text { current_solution }[i]=\text { current_solution }[i]+\mathrm{v}[i]
$$

$v[i]$ is the particle velocity, current_solution $[i]$ array holds is the current particle position. pbest and gbest are defined as stated before. $\operatorname{rand}()$ is a random number between $(0,1) . c 1, c 2$ are cognitive and social acceleration factors. Usually $c 1=c 2=2$.

\section{HAAR WAVELET TRANSFORMATION}

Haar Wavelet Transformation is a very basic transformation used to convert raw bits into wavelets[22]. The 2D Haar-DWT procedure consists of two simple operations, first one is horizontal operation and thereafter the vertical operation. The operation process is described as follows:

Step1. All the pixels are scanned from left to right in horizontal order and thereafter addition and subtraction operation is performed between the neighbour pixels. The sum is stored in the left and the difference is stored on the right side of the image. The process is repeated until all the pixels are scanned. The sum represents low frequency $(\mathrm{L})$ and difference represents high frequency $(\mathrm{H})$.

Step 2. Pixels are scanned again from top to bottom in vertical direction following the same way. Sum is stored at top and difference is stored at bottom position of the image. The process is continued till the end of pixels. 
Step 3. At the end 4 sub-bands are obtained, they are LL, HL,LH and HH respectively. This procedure is known as first order 2-D Haar wavelet transformation. Figure 1 shows the position of all 4 sub-bands in the image.

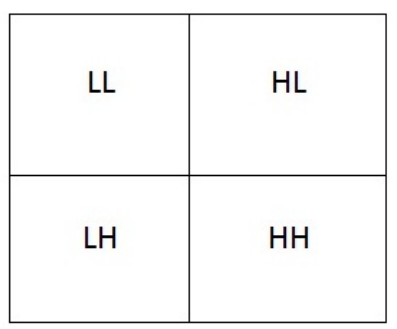

Fig 1. Position of sub-bands after performing horizontal and vertical operation.

All these 4 sub-bands are known as approximate band (LL), horizontal band (HL), vertical band (LH) and the diagonal band $(\mathrm{HH})$. The approximate band contains the most significant information of the spatial domain image and other bands contain the high frequency information such as edge details. Therefore the LL band image is quite similar with the original image. Figure 2 shows $1^{\text {st }}$ level Haar DWT transformation of Image Lena.
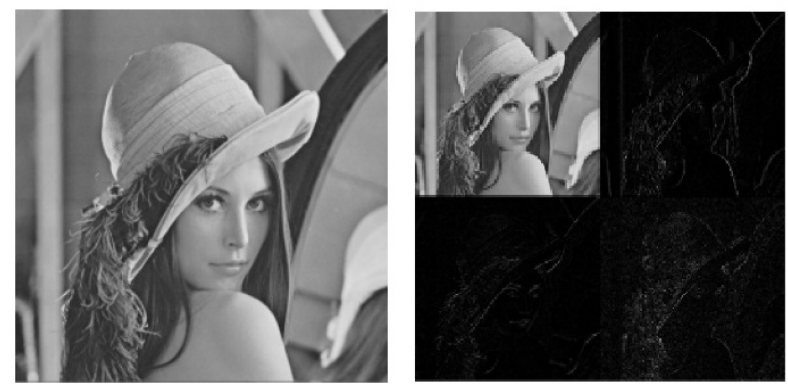

Fig 2. Original Image Lena(Left) and All 4 sub-bands after applying $1^{\text {st }}$ level Haar DWT transformation.

\section{PROPOSED WORK}

\section{A. Fitness Function:}

An appropriate fitness function has maximum influence in result. Due to embedding each and every block induce some noise. High capacity blocks have a probability of incorporating more noise. Purpose of the fitness function is to identify blocks having noise level above a certain percentage and thereafter not to account them in calculation. The noise threshold level is not fixed for all type of blocks, it depends on the number of bits embedded (capacity). The fitness function maintains the noise-capacity balance and ignores noisy blocks from calculation. PSNR between the embedded block and the original block is computed using equation (8) \& (9).

$$
\begin{aligned}
& M S E=\frac{1}{M * N} \sum_{i=0}^{N-1} \sum_{j=0}^{M-1}[X(i, j)-Y(i, j)]^{2} \\
& P S N R=10 \log _{10} \frac{L^{2}}{M S E}
\end{aligned}
$$

Here, $X$ and $Y$ are arrays of size $\mathrm{N} \times \mathrm{M}$, respectively representing the $\mathrm{Y}$-channel frame of reference (i.e. the original copy) and Y-channel frame of the encoded/impaired copy. The MSE between the two signals is defined in equation(8) and PSNR is calculated using equation(9). $L$ reflects the range of values that a pixel can take: for example, if the $Y$ channel is encoded with a depth of 8 -bit, then $L=2^{\wedge} 8-1=255$. The result is expressed in decibels $(\mathrm{db})$.

The fitness function for a block is calculated using equation (10).

$$
F=((\text { Capacity }) \times \gamma)+P S N R
$$

Here 'Capacity' is the total number of bits to be hidden within a block. $\gamma$ is the controlling factor; $\gamma$ is used to control the balance between capacity and PSNR. From experiments it is observed that the value of $\gamma$ is to be kept between 0.2 to 0.7 . Where 0.2 is lowest and 0.7 is highest value. Maintaining the value of $\gamma$ around the higher side of the prescribed range, prioritize the capacity of the block. When $\gamma$ lies near the lower side of the range, 
blocks with better PSNR value are prioritized. However, from several experiments it is observed that the value of $\gamma$ between 0.3 to 0.5 results in a stego image with balanced value of capacity and PSNR.

\begin{tabular}{|c|c|c|}
\hline Pi1 & Pi2 & Pi3 \\
\hline Pi4 & Pic & Pi5 \\
\hline Pi6 & Pi7 & Pi8 \\
\hline
\end{tabular}

Fig 3. Picture of a $3 \times 3$ block from cover image

B. Stepwise description of the proposed work:

\section{Embedding Algorithm}

Step 1. 2D DWT is applied to obtain arrays of four subbands(LL, LH,HL and HH). LH,HL and HH bands are selected for embedding.

Step 2. Randomly $n$ number of non-overlapping blocks of $3 \times 3$ are selected from the arrays containing frequency values.

Step 3. An array of secret bits, named $A[]$ is created. $S_{i}$ (starting pointer) and $E_{i}$ (ending pointer). ( $i=1,2,3 \ldots . \ldots$. and ' $i$ ' indicate the block number in sequence) is created to keep track. $S_{i}$ always points at the starting position of $A[] . E_{i}$ is $S_{i}+$ capacity of a block, i.e. the last bit position of the currently fetching bit stream. Value of $S_{i}$ and $E_{i}$ changes for each block. Secret data is equally distributed into three subbands.

Step 4. Capacity $\left(C_{i}\right)$ of $i$ th block is calculated following the embedding procedure.

Step 4.1 While embedding, PVD and LSB embedding approaches are followed. Each negative number is converted in corresponding positive number. Values exceeding the range of 255 are not considered for PVD embedding. In each block pixels are numbered, $P_{i c}$ indicates the centre bit $(\mathrm{i}=1,2 \ldots . . n), n$ is number of particle. Other pixels are represented by $P_{i l,} P_{i 2, \ldots .} P_{i \delta}$ (See figure 3). At first, from $P_{i c} 4$ LSB bits are replaced using LSB embedding technique and thereafter embedding error is adjusted using optimal pixel adjustment process (OPAP). OPAP adjustment is described as follows: $P_{i c}$ is the central pixel of a block. $P_{i c}^{\prime}$ is the pixel after embedding 4 LSB bits using LSB substitution method. $p^{\prime \prime}{ }_{i c}$ is the pixel after applying OPAP[21]. $\delta_{i}=p_{i c}^{\prime}{ }_{i c} p_{i c}$, is the embedding error between $p^{\prime}{ }_{i c}$ and $p_{i c}$. Now $p^{\prime \prime}{ }_{i c}$ is calculated using the following cases in equation(11).

Casel: $\left(2^{k-1}<\delta<2^{k}\right)$ : if pic $\geq 2^{k}$

Then $p^{\prime \prime}{ }_{i c}=p_{i c}^{\prime}-2^{k}$ Otherwise $p^{\prime \prime}{ }_{i c}=p^{\prime}{ }_{i c}$;

Case $2:\left(-2^{k-1} \leq \delta i \leq 2^{k-1}\right)$ : if $p^{\prime \prime}{ }_{i c}=p^{\prime}{ }_{i c}$;

Case $3:\left(-2^{k}<\delta i<2^{k-1}\right)$ : if $p_{i c}<256-2^{k}$, then

$p^{\prime \prime}{ }_{i c}=p^{\prime}{ }_{i c}+2^{k}$; Otherwise $p^{\prime \prime}{ }_{i c}=p^{\prime}{ }_{i c}$;

Step 4.2. Embedding using PVD is computed in this step. The difference between the central pixel $\left(P_{i c}\right)$ and other pixels are calculated one by one. Here $P_{i k}$ represents pixels other than central pixel within a block. $(k=1,2 \ldots .9)$. Secret bits are embedded within the difference gap using PVD. Here is the detail about PVD embedding technique.

After embedding new bits using PVD, both $P_{i c}$ and $P_{i k}$ is affected. In the proposed approach $P_{i c}$ remains unchanged and only $P_{i k}$ is changed. $P_{i k}^{\prime}$ is the modified pixel value after embedding secret bits between $P_{i k}$ and $P_{i c} . \delta_{i k}^{\prime}$ is the difference between pixels before embedding and after embedding secret bits.

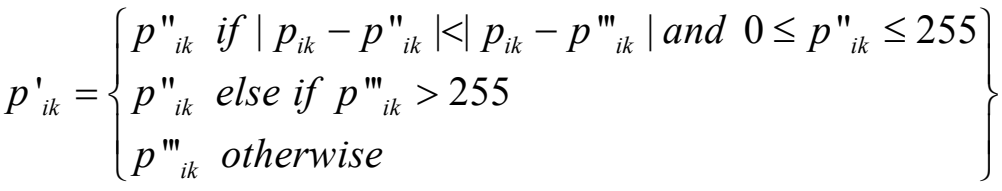

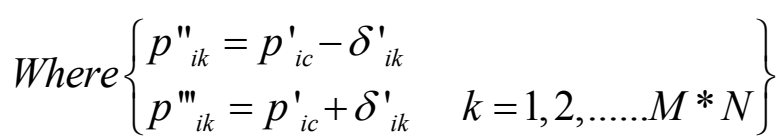


Step 5. While embedding bits from $A[]$ is fetched following the steps:

$$
\begin{gathered}
\mathrm{i}>\text { For } i=1 \text { set } S_{i}=0 \text { and } \mathrm{E}_{i}=S_{i}+\mathrm{C}_{i} \\
\mathrm{ii}>\text { Fetch } C_{i} \text { number of bits from } S[] \text { and embed them. } \\
S_{i}=S_{i-1}+1 \text { and } E_{i}=S_{i}+C_{i} \text { is upgraded for next block. }
\end{gathered}
$$

Step 6. Blocks with best fitness value are identified. (Detail about fitness function discussed in Section V) Velocity of all the blocks towards the block having best fitness value(gbest) upgraded using equation 6 and 7. Each block upgrades its personal best value(pbest) in each generation with respect to fitness function.

Step 7. Step 4 and step 5 repeated for $m$ times. (where $m$ is the number of generations/Iterations).

Step 8. Secret bits are embedded within selected blocks following the procedure already explained in step 3 and step 4.

Step 9. Inverse DWT is applied to get the stego image.

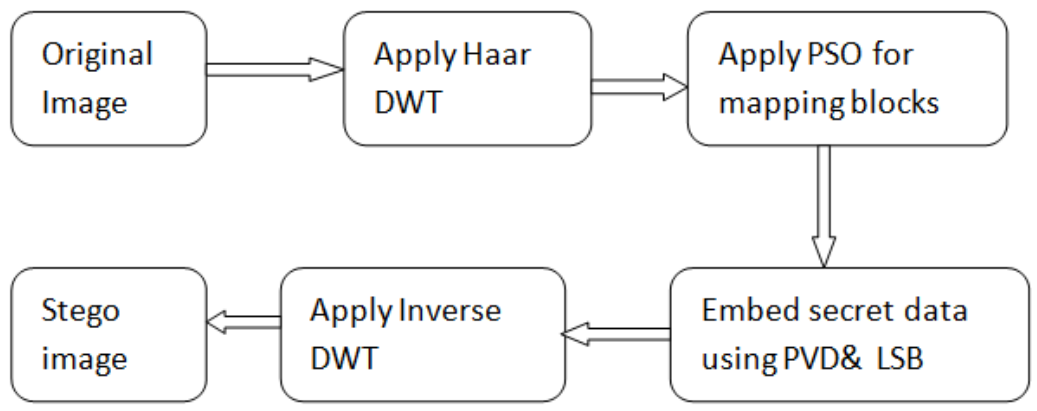

Fig 4.Diagrammatic representation of the embedding process.

\section{Extraction algorithm}

Step 1. DWT applied to obtain the subbands and selected blocks are identified to order them in a sequence.

Step 2. Pixels from the blocks are arranged in a sequence according to Figure 3. Bits are extracted using PVD following the sequence.

a $>$ While extracting, gap is calculated between middle pixel and other pixels one by one.

b $>$ Number of bits embedded using Equation 3 is calculated.

c $>$ Bits extracted using opposite procedure explained in equation 4.

Step 3. Extracted bits are arranged according to the sequence.

\section{EXPERIMENTED RESULTS}

In this section to ensure the quality of the stego image different approaches has been carried out. Usually, after embedding noise incorporated due to change in bits. To ensure that the noise level is within the acceptable range, PSNR and SSIM is used for quality measurement standard. PSNR value calculation process is already described using equation (8)\&(9) in section IV.

MSSIM (Mean structural similarity index) is a standard image quality measurement technique granted by Universal image quality index (UQI) [20]. MSSIM could be calculated using equation (13)

$$
M S S I M=\frac{1}{M} \sum_{j=1}^{M} \operatorname{SSIM}\left(x_{j}, y_{j}\right)
$$

Where $x_{j}$ and $y_{j}$ are the image contents of the $j_{t h}$ local window and $M$ is the number of local windows in the image. SSIM calculation process is described in equation (14).

$$
\operatorname{SSIM}(x, y)=\left(2 x^{\prime} y^{\prime}+c_{1}\right)\left(2 \sigma_{x y}+c_{2}\right) /\left(x^{\prime 2}+y^{\prime 2}+1\right)\left(\sigma_{x}^{2}+\sigma_{y}^{2}+c_{2}\right)
$$

Where $x$ and $y$ are corresponding windows of the same size of the original and stego image. $x^{\prime}$ and $y^{\prime}$ are the corresponding averages of $x$ and $y$ respectively. $\sigma_{x}^{2}$ and $\sigma_{y}^{2}$ are the corresponding variances of $x$ and $y . \sigma_{x y}$ is the covariance of $x$ and $y . c 1$ and $c 2$ are constants with appropriate value.

The proposed algorithm along with other existing algorithms has been implemented in Matlab programming language in a personal computer with Intel Core 2 Duo processor having 2 GB of memory. Table I,II and III shows the capacity (Total amount of bits embedded), PSNR and MSSIM value observed using the proposed method. Here value of $\gamma=0.4$. Number of generation is 50 . All the greyscale images used in experiment are of size $512 \times 512$. 
Table I. PSNR, Capacity and MSSIM observed using proposed algorithm in LH,HH \& LH subbands.

\begin{tabular}{|l|l|l|l|l|l|l|l|}
\hline \multirow{2}{*}{ Image } & \multirow{2}{*}{ PSNR } & \multicolumn{2}{|c|}{ LH } & \multicolumn{2}{c|}{ HH } & \multicolumn{2}{c|}{ LH } \\
\cline { 3 - 8 } & & Capacity & PSNR & Capacity & PSNR & Capacity & PSNR \\
\hline Lena & 52.68 & 8842 & 48.83 & 8586 & 47.94 & 8849 & 48.95 \\
\hline Baboon & 50.04 & 9235 & 47,25 & 9539 & 47.11 & 9723 & 47.15 \\
\hline Peppers & 52.26 & 8073 & 47.53 & 8021 & 47.83 & 8489 & 47.37 \\
\hline Tiffany & 51.51 & 8035 & 47.32 & 8920 & 47.73 & 8537 & 47.31 \\
\hline Aerial & 52.73 & 8356 & 48.37 & 8739 & 47.38 & 8635 & 47.25 \\
\hline Goldhill & 51.63 & 8016 & 47.83 & 8047 & 47.35 & 8342 & 47.35 \\
\hline Boat & 51.94 & 7093 & 47.57 & 8063 & 47.58 & 8545 & 47.35 \\
\hline Brian & 52.63 & 8763 & 48.83 & 8674 & 48.58 & 8546 & 48.64 \\
\hline
\end{tabular}

In Table I PSNR observed using the proposed algorithm and PSNR for individual subbands(LH,HH \& LH) is show. In Table II PSNR,MSSIM and capacity of entire image is shown. PSNR is shown in db and capacity is calculated in bits.

Table II. Shows PSNR(db) capacity and MSSIM observed for the entire image.

\begin{tabular}{|l|l|l|l|}
\hline Image & PSNR & Capacity(in bits) & MSSIM \\
\hline Lena & 46.21 & 75385 & 0.9977 \\
\hline Baboon & 43.54 & 79649 & 0.9957 \\
\hline Peppers & 45.63 & 75576 & 0.9883 \\
\hline Tiffany & 45.73 & 75896 & 0.9951 \\
\hline Aerial & 46.74 & 75642 & 0.9870 \\
\hline Goldhill & 44.38 & 74903 & 0.9930 \\
\hline Boat & 45.52 & 75458 & 0.9537 \\
\hline Brian & 46.32 & 75328 & 0.9826 \\
\hline
\end{tabular}

Table III. Contains PSNR and capacity observed from experiments proposed by Fan Li et.al [18],and the proposed method.

\begin{tabular}{|l|c|c|c|c|}
\hline \multirow{2}{*}{} & \multicolumn{2}{|c|}{ Fan Li et al.[18] } & \multicolumn{2}{c|}{ Proposed method } \\
\cline { 2 - 5 } & Capaity (Bits) & PSNR & Capaity (Bits) & PSNR \\
\hline Lena & 23007 & 52.47 & 25864 & 52.83 \\
\hline Baboon & 24865 & 50.33 & 28497 & 50.04 \\
\hline Peppers & 23854 & 52.03 & 24543 & 52.26 \\
\hline Tiffany & 24006 & 51.83 & 25492 & 51.51 \\
\hline Brian & 23411 & 52.41 & 25983 & 52.63 \\
\hline
\end{tabular}

In Table III. The proposed method is compared with the method proposed by Fan Li et al.[18].

While comparison both PSNR, capacity and MSSIM is compared. All the cover image used for experiment is $512 \times 512$ greyscale image. Figure 4 . Shows standard images of size $512 \times 512$ used in the experiments. Figure 5 shows images after embedding. 
a.

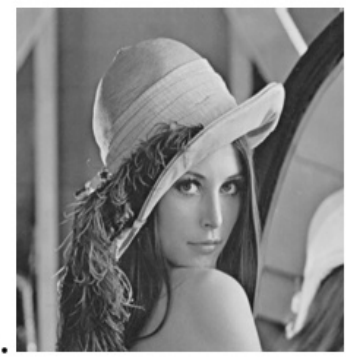

b.
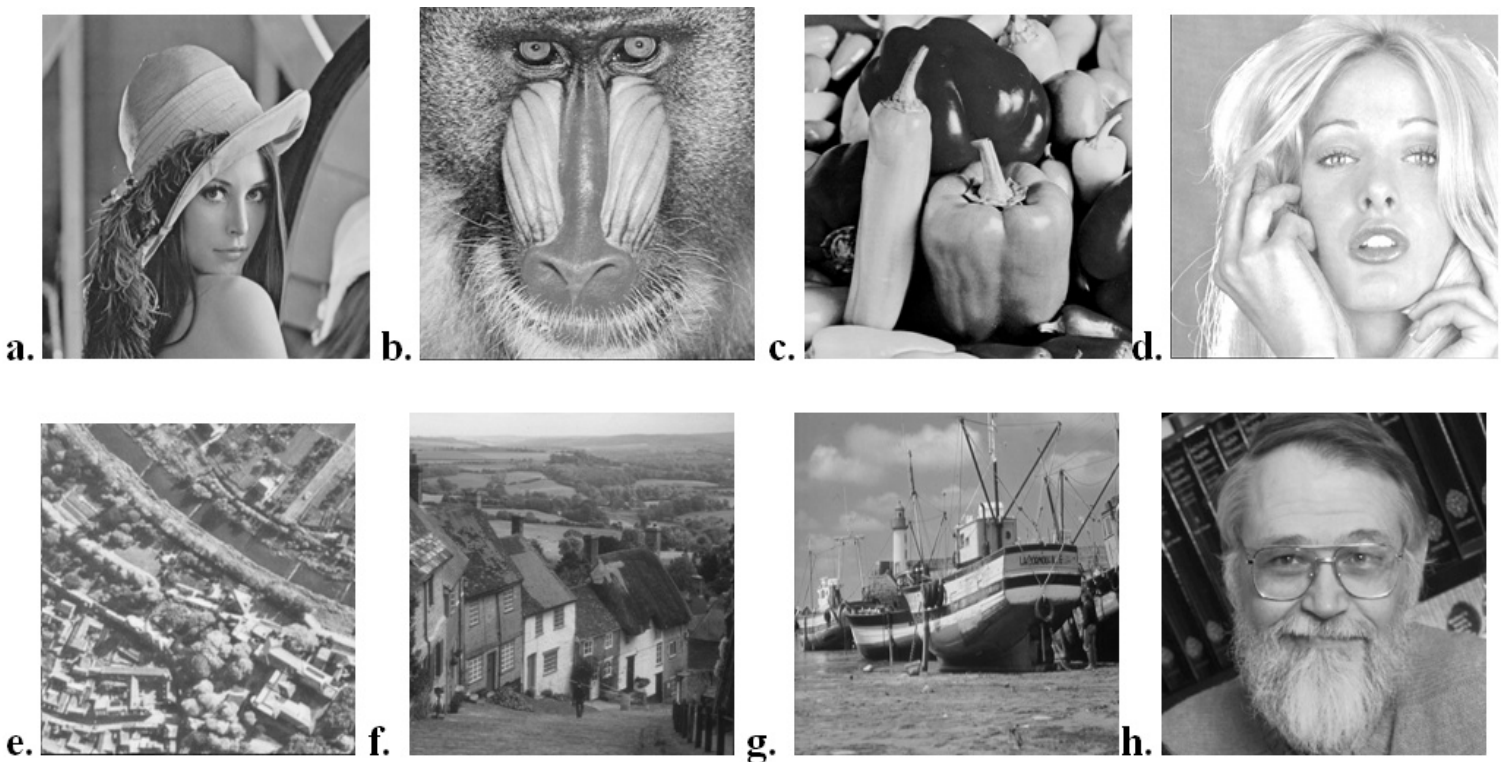

Fig 4. Original image a. Lena b. Baboon c. Peppers d. Tiffany e. Aerial f. Goldhill g. Boat h. Brian.
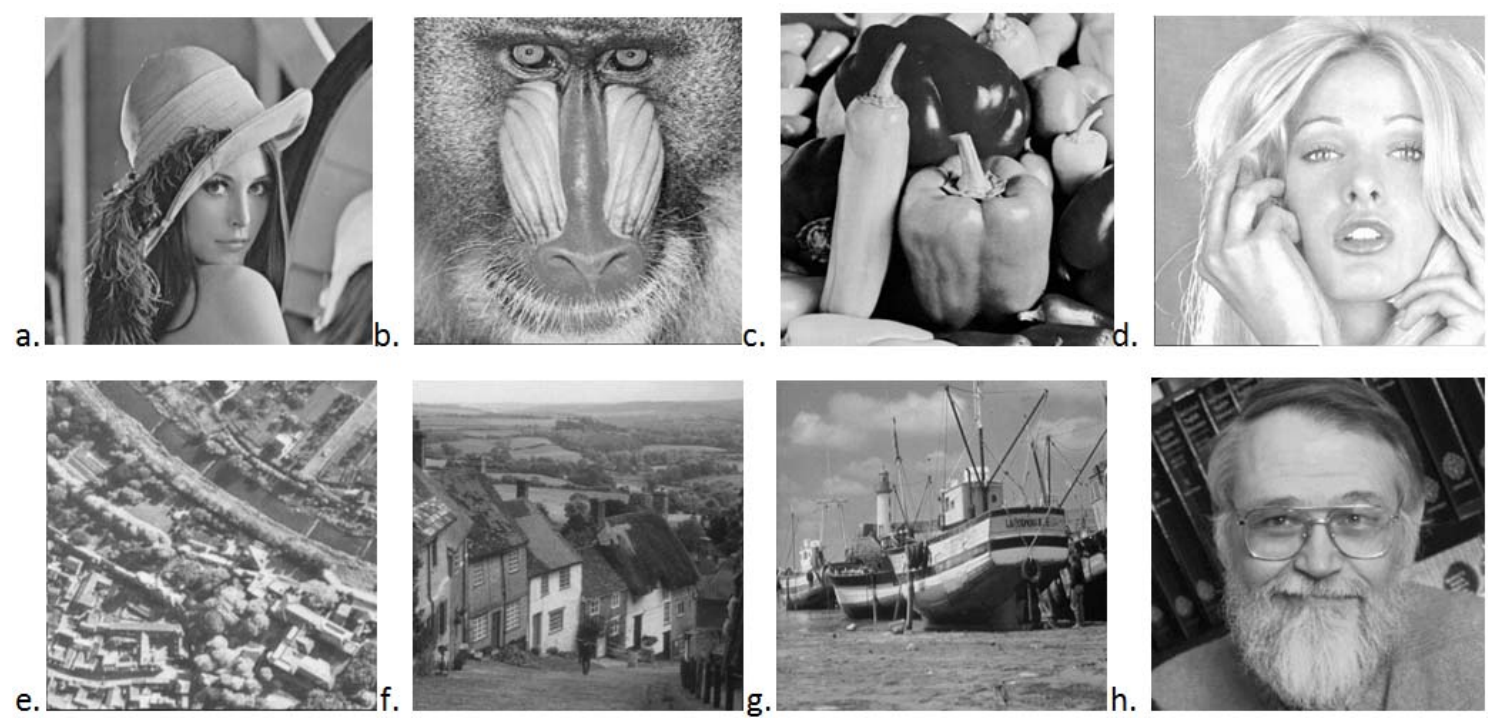

Fig 5. Images after embedding secret data (Stego image). a. Lena and b.Baboon. C.Pepers d.Tiffany e.Aerial f.Goldhill g.Boat and h.Brian .

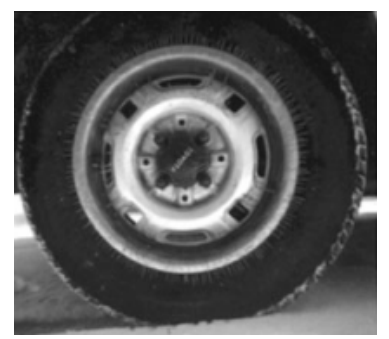

Fig 6. Secret Image tyre.pgm

\section{RESULT ANALYSIS}

In this section result obtained from different experiment are analysed. Table I shows subband wise PSNR values along with total number of bits embedded, obtained from the proposed experiment. Eight different greyscale images are used as cover image. Table II shows that the proposed experiment is also stable for large capacity image. In Table III results from the proposed experiment are compared with existing methods. Hiding capacity observed in table III using the proposed approach is higher than Fan Li's [18] method. At the same time PSNR observed is either greater or almost same. From here it is sure that stego images using proposed method includes less noise when compared with the existing algorithms. Another quality measurement parameter MSSIM also ensures that the level of noise after embedding is negligible(shown in Table II). Boundary regions are manipulated so that none of the values falls out of range. We may observe the visual difference figure 4 and 
figure 5 between the original images and stego image. Henceforth, it is obvious that assuming the presence of secret data is almost impossible.

\section{CONCLUSION}

In this paper an adaptive data hiding technique based on LSB substitution, OPAP and PVD has been proposed. Efficient utilisation of PSO in the proposed approach use only selected areas where greyscale intensity value varies between pixels in a maximum range. Due to the reason secret bits are not spread over those areas in the cover image which are sensitive to change. Inclusion of both PSNR and capacity in fitness function helps to improve selection of quality blocks. While considering only one parameter (capacity/ quality, i.e. good PSNR value) in fitness function choose blocks of a particular category and ignore the other. When choosing blocks, the controlling factor $(\gamma)$ helps to ignore biased blocks, i.e. blocks, biased towards a particular parameter are excluded. Altering frequency values in LH,HH and HL subbands and ignoring LL subband effect the image in minimum sense. From the result section it is obvious that the proposed algorithm produces stego image having less noise. In future our scope would be to increase capacity and image quality of the proposed algorithm. This idea could be used to protect any digital document against vulnerable attack \& could raise high the acceptability of this novel E-commerce application.

\section{REFERENCES}

[1] T.Sharp, An implementation of key-based digital signal steganography. In: Proc. Information Hiding Workshop. Volume 2137 of Springer LNCS. 2001, 13-26.

[2] Niel F. Johnson, Z.Duric, S.Jajodia, "Information Hiding, and Watermarking - Attacks \& Countermeasures," Kluwer Academic Publishers.2000.

[3] C.K.Chan, L. M. Cheng, (2004), "Hiding data in images by simple LSB substitution". Pattern Recognition Vol. 37, p. 469-474.

[4] D.C. Wu W.H.Tsai, 2003. "A steganographic method for images by pixel-value differencing". Pattern Recognition Letters 24, 9-10, $1613-1626$.

[5] Ko-Chin Chang, Chien-Ping Chang, Ping S. Huang, Te-MingTu. “.A Novel Image Steganographic Method Using Tri-way Pixel-Value Differencing."Journal of Multimedia. 2008.

[6] M. Khodaei and K. Faez, "New adaptive steganographic method using least-significant-bit substitution and pixel-value differencing" IET Image Processing, Vol. 6, pp. 677-686, 2012.

[7] Yuan-Yu Tsai, Jian-Ting Chen, and Chi-Shiang Chan "Exploring LSB Substitution and Pixel-value Differencing for Block-based Adaptive Data Hiding". International Journal of Network Security, Volume.16, No.6, PP.530-535, Nov. 2014

[8] (In press)T.Halder, S.Karforma "A Block-based Adaptive Data Hiding Approach using Pixel-Value-Difference and LSB Substitution to Secure E-Governance Documents". Journal of Information Processing System, Korea.

[9] Wang RZ, Lin CF, Lin JC. "Image hiding by optimal LSB substitution and genetic algorithm”. Pattern Recognit 2001;34:671-713.

[10] M. Khodaei, K. Faez "Image Hiding by using genetic algorithm and LSB substitution". In: Proc ICISP 2010. LNCS 6134. p. 404-11.

[11] P. Bedi, R.Bansal, P.Sehgal. "Using PSO in image hiding scheme based on LSB substitution". In: Proc international conference on advances in computing and communications (ACC 2011); CCIS 192; 2011. p. 259-68.

[12] F.Heriniaina, \& J.Sang, "Using PSO algorithm for simple LSB substitution based steganography scheme in DCT transformation domain". In Proceedings of the Second international conference on advances in swarm intelligence. Springer-Verlag, 2011.

[13] P.Bedi, R.Bansal, P.Sehgal. "Using PSO in a spatial domain based image hiding scheme with distortion tolerance". Computers and Electrical Engineering, 2013. 640-654.

[14] A. K. Gulve and M. S. Joshi, "An image steganography algorithm with five pixel pair differencing and gray code conversion," International Journal of Image, Graphics and Signal Processing, vol. 6, no. 3, pp. 12-20, 2014.

[15] E. Ghasemi, J. Shanbehzadeh, and N. Fassihi, "High capacity image steganography using wavelet transform and genetic algorithm," in Proceedings of the International Multi Conference of Engineers and Computer Scientists (IMECS '11), pp. 495-498,Hong Kong, March 2011.

[16] A. K. Al-Asmari, M. A. Al-Qodah, and A. S. Salama, "Waveletpixel value differencing technique for digital images data hiding," in Proceedings of the IEEE International Conference on System Engineering and Technology (ICSET '11), pp. 15-18, June 2011.

[17] A.K.Gulve, M.S.Joshi, “An Image Steganography Method Hiding Secret Data into Coefficients of Integer Wavelet Transform Using Pixel Value Differencing Approach,”. Mathematical Problems in Engineering. Hindawi Publishing Corporation. 2015.

[18] F.Li, Q.Mao and C.C.Chang, " Reversible data hiding scheme based on the Haar discrete wavelet transform and interleaving prediction method”. Multimedia Tools Appl. Springer Science+Business Media New York 2017.

[19] J.Kennedy, , \& R.Eberhart, "Particle Swarm Optimization". Paper presented in the IEEE International Conference on Neural Networks IV. doi:10.1109/ICNN.1995.488968

[20] Z.Wang , A.C.Bovik. “A universal image quality index,” IEEE Signal Process Lett 2004;9(3):84-94.

[21] C. K. Chan and L. M. Cheng, "Hiding data in images by simple LSB substitution,"” Pattern Recognition, vol. 37, no. 3, pp. 469-474, 2004.

[22] A. R. Calderbank, I. Daubechies, W. Sweldens, and B.-L. Yeo,"Wavelet transforms that map integers to integers," Applied and Computational Harmonic Analysis, vol. 5, no. 3, pp. 332-369,1998. 


\section{AUTHOR PROFILE}
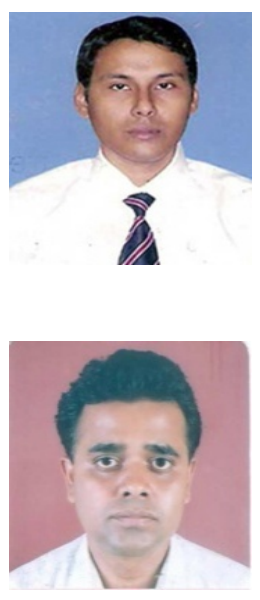

Mr.Tanmoy Halder has completed his BCA(Bachelor in Computer Application) from the University of Burdwan in 2004 and MCA(Master in Computer Application) from The same university in 2007. Now he is working as Assistant Professor in Dr.B.C.Roy Engineering College, Durgapur, India. His research area is network security using Steganography. He has been working in this area since last 4 years and have five publications in International journal and conferences.

Dr. Sunil Karforma has completed B.E. (Computer Science and Engineering) and M. E. (Computer Science and Engineering) from Jadavpur University. He has completed Ph. D. in the field of Cryptography. He is presently holding the post of Professor and the Head of the Department in the Department of Computer Science, The University of Burdwan. Network security and e-commerce is his field of interest in research area. He has published approximately 16 research papers in reputed National and International journals and proceedings. 